\title{
Antinociceptive effects of topical mepivacaine in a rat model of HIV-associated peripheral neuropathic pain
}

This article was published in the following Dove Press journal:

Journal of Pain Research

6 June 2016

Number of times this article has been viewed

\section{Jacqueline Sagen \\ Daniel A Castellanos ${ }^{\dagger}$ \\ Aldric T Hama}

The Miami Project to Cure Paralysis, University of Miami Miller School of

Medicine, Miami, FL, USA

tDaniel A Castellanos passed away on April 14, 2010
Correspondence: Jacqueline Sagen The Miami Project to Cure Paralysis, University of Miami Miller School of Medicine, 1095 Northwest 14th Terrace, Miami, FL 33134, USA

Tel +I 3052435618

Fax + I 3052436038

Email jsagen@miami.edu
Background: A consequence of HIV infection is sensory neuropathy, a debilitating condition that degrades the quality of life of HIV patients. Furthermore, life-extending antiretroviral treatment may exacerbate HIV sensory neuropathy. Analgesics that relieve other neuropathic pains show little or no efficacy in ameliorating HIV sensory neuropathy. Thus, there is a need for analgesics for people with this particular pain. While lidocaine is used in the management of painful peripheral neuropathies, another local anesthetic mepivacaine, with a potentially improved bioavailability, could be utilized for the management of HIV neuropathic pain.

Methods: The efficacy of topical anesthetics was evaluated in a preclinical rodent model of painful peripheral neuropathy induced by epineural administration of the HIV envelope protein gp120 delivered using saturated oxidized cellulose implanted around the sciatic nerve. Beginning at 2 weeks following gp120 administration, the effects of local anesthetics topically applied via gauze pads were tested on heat and mechanical hyperalgesia in the hind paw. Rats were tested using several concentrations of mepivacaine or lidocaine during the following 2 weeks. Results: By 2 weeks following epineural gp120 implantation, the ipsilateral hind paw developed significant hypersensitivity to noxious pressure and heat hyperalgesia. A short-lasting, concentration-dependent amelioration of pressure and heat hyperalgesia was observed following topical application of mepivacaine to the ipsilateral plantar hind paw. By contrast, topical lidocaine ameliorated heat hyperalgesia in a concentration-dependent manner but not pressure hyperalgesia. Equipotent concentrations of mepivacaine and lidocaine applied topically to the tail of mice significantly increased tail withdrawal latencies in the tail flick test, demonstrating that both local anesthetics attenuate responding to a brief noxious stimulus.

Conclusion: These findings showed that mepivacaine, rather than lidocaine, consistently attenuated two distinct symptoms of neuropathic pain and suggest that topical formulations of this local anesthetic could have utility in the alleviation of clinical HIV neuropathic pain.

Keywords: chronic pain, acute pain, analgesia, AIDs-related pain, distal sensory neuropathy, local anesthetics

\section{Introduction}

Distal sensory polyneuropathies are the most common and disabling neurological consequence of HIV infection. Symptoms of HIV sensory neuropathies include numbness and sensory loss but are frequently accompanied by persistent neuropathic pain which can significantly diminish the quality of life and daily functioning of these patients. ${ }^{1-6}$ HIV neuropathic pain is reminiscent of other neuropathic pains such as painful diabetic neuropathy, which is characterized by burning sensations, sharp stabbing, and paresthesias, predominantly affecting the distal innervation of the feet and hands. 
While there are a number of treatments that have been used in managing other peripheral neuropathic pains, including anticonvulsants such as pregabalin and antidepressants such as amitriptyline, none of these have demonstrated significant efficacy in HIV neuropathic pain. . $^{5-10}$

Topical local anesthetics, such as lidocaine, are recommended as a first-line therapy in postherpetic neuralgia and other localized neuropathic pains. ${ }^{11,12}$ There are significant benefits in using topical anesthetics in pain management, such as use on an as-needed basis rather than adherence to a fixed dosing schedule for parenteral medications and safety. With regard to painful HIV-associated neuropathy, there are no available data from placebo-controlled randomized clinical trials demonstrating the efficacy of topically applied local anesthetics. Indeed, in the only available placebo-controlled randomized clinical trial of topical lidocaine, there were no significant differences in efficacy from placebo. ${ }^{13}$

Mepivacaine, a structural analog of bupivacaine with distinct pharmacokinetics, ${ }^{14}$ could be more suitable for distal neuropathies, such as painful diabetic neuropathy and HIV neuropathy, which primarily involves the feet, in contrast to postherpetic neuralgia which involves more proximal areas such as the thorax. Mepivacaine has improved bioavailability due to its intrinsic vasoconstrictor effect, reducing the rate at which the drug is cleared away from the site of application. In addition, mepivacaine may have improved neurological safety, as it showed the least neurotoxic effects on developing or regenerating primary cultured neurons among a series of local anesthetics tested, with lidocaine having the highest neurotoxic potential. ${ }^{15}$ Mepivacaine has been used clinically for intraganglionic nerve blocks ${ }^{16}$ but has not been tested for topical application in neuropathic pain.

A preclinical model of HIV-induced neuropathy has been previously described which demonstrates robust signs of neuropathic pain. ${ }^{17,18}$ Although the pathogenic mechanisms underlying HIV-associated neuropathies are poorly understood, direct involvement of viral proteins such as gp120 in producing neurotoxicity and axonal degeneration has been suggested by in vitro and in vivo studies. ${ }^{19-24}$ Epineural placement of gp120 around the sciatic nerve results in an inflammatory response in the peripheral nerve and subsequent development of increased responsiveness of the hind paw to noxious stimuli ("hyperalgesia"). A number of clinical analgesics have been tested in this model, but the efficacy of a topical local anesthetic has not been evaluated. ${ }^{18}$ Thus, the current study evaluated the antinociceptive effects of topically applied mepivacaine in a rat model of HIV-associated peripheral neuropathic pain. Portions of this work were presented previously as a poster at scientific conferences. ${ }^{25,26}$

\section{Methods}

\section{Animals}

To evaluate the effects of topical anesthetics in a model of HIV-associated neuropathic pain, male Sprague Dawley rats (Harlan Sprague Dawley Inc., Indianapolis, IN, USA) weighing 150-200 g at delivery were used. For assessment of anesthetic effects following topical application to normal skin using the radiant tail flick assay, male mice (C57BL/6; Charles River Laboratories, Wilmington, MA, USA) weighing 15-20 g at delivery were used. Animals were housed in a temperature-controlled environment $\left(22^{\circ} \mathrm{C} \pm 1{ }^{\circ} \mathrm{C}, 30 \%-55 \%\right.$ humidity) under a 12 -hour light/ dark cycle two animals per cage (rats) or five animals per cage (mice). Food and water were available ad libitum. Animals were acclimatized to the housing facility for at least 1 week prior to the start of the studies. All animal procedures followed National Institutes of Health guidelines concerning the care and use of laboratory animals and were reviewed and approved by the University of Miami Animal Care and Use Committee.

\section{Rat gP I 20 HIV-associated neuropathic pain model}

The rat gp120 model was induced by application of HIV envelope protein gp120 to the sciatic nerve; the virus itself was not utilized. ${ }^{17}$ Under isoflurane anesthesia, the left sciatic nerve was isolated under aseptic surgical conditions by blunt dissection of the biceps femoris, without damaging the perineurium. Oxidized cellulose $\left(\mathrm{Oxycel}^{\circledR}\right.$; BD, Franklin Lakes, NJ, USA) was used as a carrier matrix to deliver gp120 directly to the sciatic nerve. Oxycel was prepared in strips of $\sim 8 \mathrm{~mm}$ length and $4 \mathrm{~mm}$ width, and saturated with a $50 \mu \mathrm{L}$ solution containing $300 \mathrm{ng}$ gp120 in water (HIV-1 MN recombinant [Baculovirus], Product 1021-2; Immunodiagnostics, Inc., Bedford, MA, USA). The Oxycel was wrapped loosely around the sciatic nerve $2-3 \mathrm{~mm}$ proximal to the trifurcation using care not to cause any nerve constriction. Following Oxycel implantation, the muscular layer was closed using 3-0 silk, and the skin sealed with veterinarian-grade cyanoacrylate. Animals were held in recovery for observation for $\sim 24$ hours post-surgery and then returned to their home cages and checked daily. These procedures resulted in the development of neuropathic pain symptoms (mechanical and thermal hyperalgesia and allodynia) by $\sim 2$ weeks following gp120 application. ${ }^{17}$ 


\section{Behavioral testing}

Rat gp 120 hyperalgesia

In rats, behavioral testing was conducted before and 2 weeks after gp120 administration to determine baseline responses prior to local anesthetic administration. For assessment of pressure hyperalgesia, responsiveness to an increasing force (measured in grams) applied to the plantar hind paw was measured with a Randall-Selitto apparatus (Ugo-Basile SRL, Monvalle, Italy). The rat was loosely wrapped in a towel, and the hind paw placed on the apparatus' pedestal. A plinth was gently lowered onto the hind paw, and the apparatus activated. A gradually increasing force $(32 \mathrm{~g} / \mathrm{s})$ was applied until the rat withdrew its paw from the apparatus. The presurgical withdrawal threshold of naïve rats was $\sim 175 \mathrm{~g}$, and a cut-off of $500 \mathrm{~g}$ was assigned in the absence of a response. For assessment of thermal hyperalgesia, responsiveness to a brief, noxious heat stimulus was measured using an apparatus previously described ${ }^{27}$ Rats were placed on an elevated glass surface and allowed to acclimate for $\sim 15$ minutes prior to baseline testing. An infrared emitter was positioned beneath one of the paws. The length of time between initiation of the stimulus and withdrawal of the rat's hind paw from the stimulus was recorded by the apparatus (measured in seconds). The stimulus intensity was adjusted to result in mean presurgical baseline latencies of 10-12 seconds, with a cut-off of 20 seconds in the absence of a response used to avoid skin damage. Because of the short duration of the anesthetic, the response to the infrared stimulus was tested once.

\section{Mouse tail flick test}

The gp120 model was used to study the potential beneficial effects of topical anesthetic treatment of neuropathic pain resulting from HIV sensory neuropathy. However, it is important to compare topical anesthetic effects in a model of sensitivity to acute pain in non-injured animals commonly used for antinociceptive testing. Toward this end, the mouse tail flick assay was selected as a simple screen for antinociceptive effects to noxious thermal stimulation.

In the mice, responsiveness of the tail to brief, noxious heat stimulation was determined using a Tail Flick Analgesia Meter (Model 33; IITC, Inc., Woodland Hills, CA, USA; set at sensitivity 7.5 and beam 5.0). Tail flick latencies (in seconds) were measured on the distal $3 \mathrm{~cm}$ region of the tail at baseline by applying a focused beam of light (thermal stimulus) to the ventral aspect of the tail. The tail flick latency is the time interval between onset of the heat stimulus and withdrawal of the tail from the beam. A maximum cut-off latency of 8 seconds was used to prevent tissue damage.
For assessment of local anesthetic effects on normal skin, treatments were administered topically to the mouse tail by immersion of the distal portion of the tail $(3 \mathrm{~cm})$ in drug or vehicle solutions for 5 minutes. Tail flick latencies were determined immediately after removal of the tail from the solution and again at 5 and 10 minutes. Testing of the drugs was done in a pseudo-Latin square design by an observer blinded to treatment assignment.

\section{Local topical anesthetics}

Pharmaceutical-grade mepivacaine base was procured from Cambrex Karlskoga AB (Karlskoga, Sweden). The local anesthetic lidocaine was selected as a "gold standard" comparator, since this agent is clinically utilized as a topical analgesic. ${ }^{11,12,28,29}$ Lidocaine base (American International Chemical, Inc., Framingham, MA, USA), manufactured by Gufic Biosciences (Mumbai, India) (Lot No. 1049), was provided by Relmada Therapeutics, Inc. (New York, NY, USA). Dimethyl sulfoxide was procured from Sigma-Aldrich Co. (St Louis, MO, USA). Fresh drug solutions were prepared on each test day. On the day of testing, dimethyl sulfoxide was diluted with distilled water to prepare a $90 \%: 10 \%(\mathrm{v} / \mathrm{v})$ solution to serve as the vehicle. Since the purpose of the study was to compare potency of topical mepivacaine with more commonly used clinical local anesthetic lidocaine, a wide range of concentrations of both agents was used. Drugs were serially diluted in vehicle and mixed by vortexing, to achieve concentrations ranging from 2 to $32 \mathrm{mM}$.

\section{Application of topical anesthetics to the hind paw of the rat gp 120 model}

For local anesthetic delivery to the rat hind paw in the region where noxious stimuli were to be delivered, a $2 \times 2$ in gauze pad was saturated with either drug or vehicle and secured to the left plantar hind paw with Parafilm for 5 minutes. Following removal of the pad, rats were immediately tested. Rats were tested again at 5 and 10 minutes after removal of the pad.

Due to the rapid onset and short duration of topical application of local anesthetic agents, animals were evaluated for thermal or mechanical responses in separate groups. In order to minimize the number of animals, rats were tested for responses to several drugs/concentrations over the 2-week period following induction of neuropathic pain, with at least 48-hour intervals between testing days. Drug dosing was done using pseudo-Latin square design by an observer blinded to treatment assignment, and continued until $n=6-9$ animals were accrued for each drug concentration and pain test (each animal was used four to five times). Although each ani- 
mal did not receive all possible treatments, all concentrations were given to at least one animal on each test day in order to minimize effects of day-to-day environmental variables. The continued presence of hyperalgesia in the rats was confirmed by predrug application baseline testing on each test day.

\section{Application of topical anesthetic to the mouse tail}

For this part of the study, 4 and $10 \mathrm{mM}$ lidocaine concentrations (based on the effective concentration range of this agent determined using the mouse tail flick test $\mathrm{t}^{30}$ ) were compared with higher concentrations of mepivacaine (8, 16, and $32 \mathrm{mM})$.

\section{Statistical analysis}

Statistical comparisons for changes in thermal and mechanical hyperalgesia in the gp120 model and mouse tail flick latencies were performed using two-way analysis of variance (repeated measures) and the Newman-Keuls test for multiple post hoc analysis (SigmaPlot 12.0). The data were also analyzed as a percent change in baseline response:

$\%$ change from baseline $=$

(drug effect - predrug effect)/predrug effect $\times 100$, (1)

wherein "drug effect" = withdrawal threshold (in grams) or withdrawal latency (in seconds) following drug or vehicle treatment, and "predrug effect" = withdrawal threshold (in grams) or withdrawal latency (in seconds) prior to drug or vehicle treatment. Thus, a $100 \%$ change is antinociceptive, whereas a $0 \%$ change indicates no efficacy. The percent change, withdrawal threshold, and latency data were presented as mean \pm standard error of the mean.

Since a reduction in symptom severity by $30 \%-50 \%$ is considered a clinically relevant therapeutic outcome in the management of neuropathic pain, "analgesia" was defined quantally as a response $\geq 33 \%$ increase over baseline calculated for an individual animal. ${ }^{2,5}$ The percentage of all rats that demonstrated such a response is presented. Data are graphically represented using GraphPad Prism software. Statistical significance was declared if the two-sided $P$-value was $<0.05$.

\section{Results}

\section{Effects of topical mepivacaine on gp I20- induced neuropathic hyperalgesia}

Baseline responses to noxious pressure (Figure 1A) and noxious heat (Figure 2A) are shown prior to gp120 surgery ("pre-gp120") and at 2 weeks following gp120 sciatic nerve exposure ("predrug"). Neither pre-gp120 nor predrug scores differed between any of the treatment groups ( $P>0.05$ at these test points, for both mechanical and heat tests). Epineural placement of gp120 around the rat sciatic nerve via Oxycel carrier resulted in significantly decreased thresholds of withdrawal from pressure (Figure 1A) and decreased latencies to withdrawal from noxious heat (Figure 2A), suggesting the

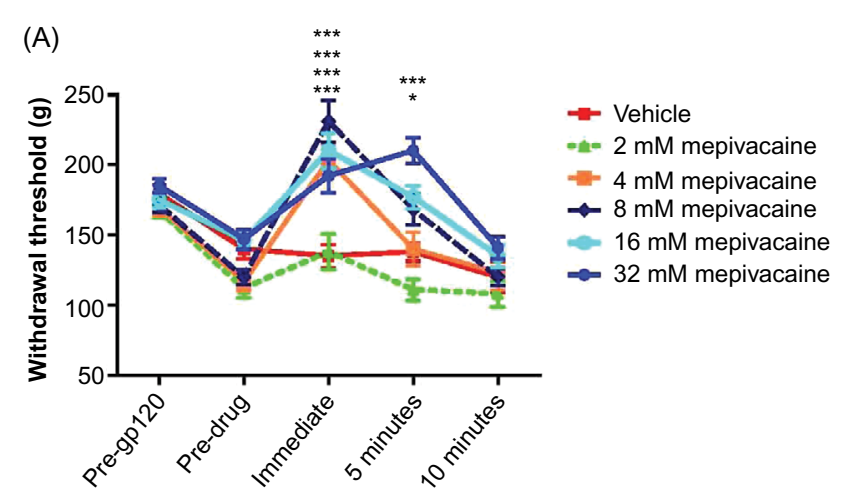

(B)
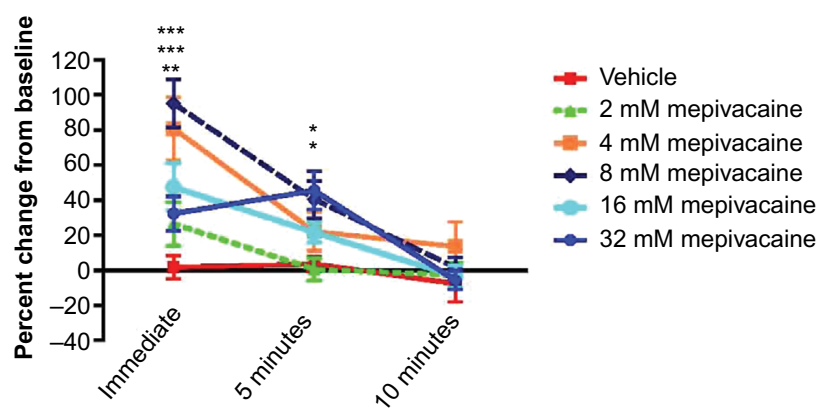

(C)

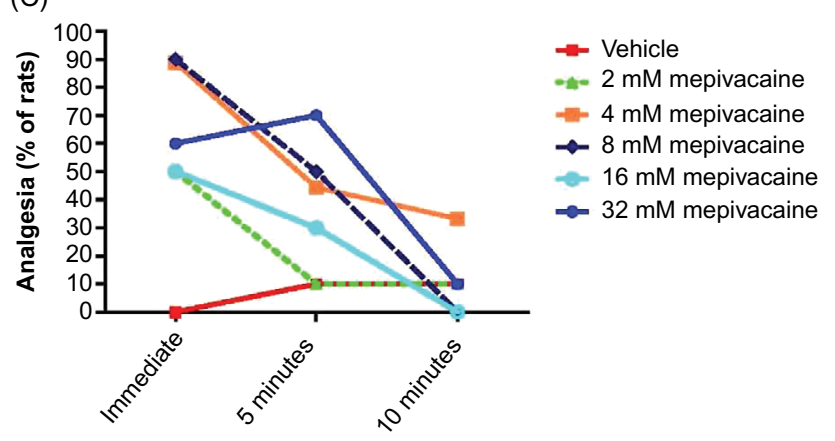

Figure I Effect of topically applied mepivacaine on mechanical hyperalgesia in rats with gp I 20-induced neuropathic pain.

Notes: (A) Effects of several concentrations (in millimolars) of topical mepivacaine or vehicle on hind paw withdrawal thresholds (in grams). Withdrawal thresholds before application of gp/20 to the sciatic nerve ("pre-gp/20") and 2 weeks after, prior to topical application ("predrug"), were measured. Rats were tested within I minute ("immediate") of removal of the gauze pad, and 5 and 10 minutes following removal of the gauze pad. Data are presented as mean \pm standard error of the mean ( $\mathrm{n}=7-10$ animals per treatment group). (B) Percent changes in mechanical withdrawal thresholds following topical mepivacaine treatment, calculated from predrug baseline scores in (A). (C) Percent of animals in each concentration group reaching $\geq 33 \%$ increase in mechanical withdrawal threshold from predrug baselines. $* P<0.05, * * P<0.01$, and $* * * P<0.001$ vs vehicle-treated rats. 
(A)

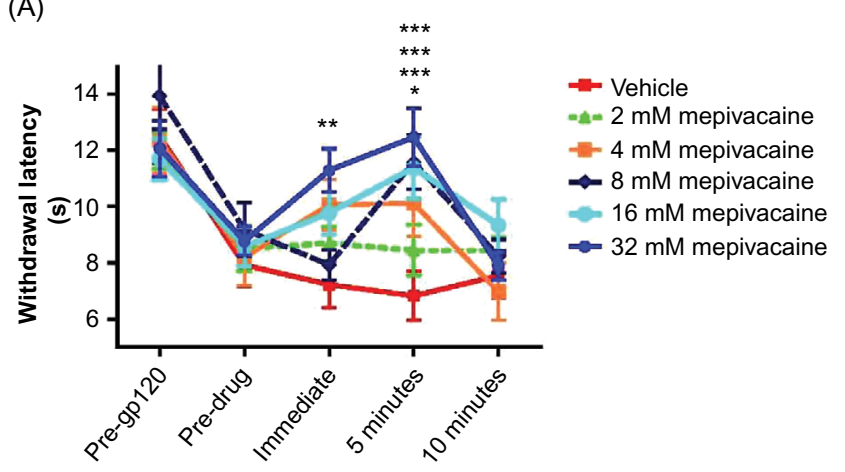

(B)

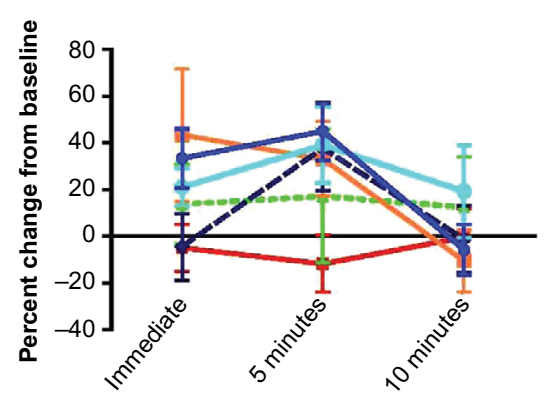

(C)

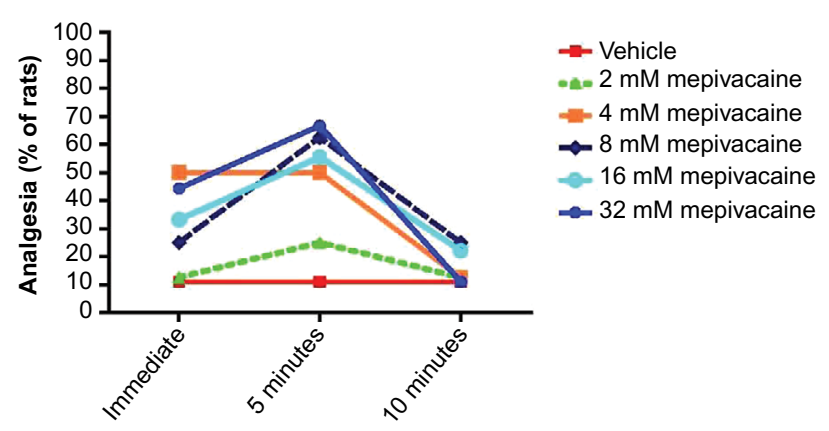

Figure 2 Effect of topically applied mepivacaine on thermal hyperalgesia in rats with gPl20-induced neuropathic pain.

Notes: (A) Effects of several concentrations (in millimolars) of topical mepivacaine or vehicle on hind paw withdrawal latencies (in seconds). Withdrawal latencies before application of gp/20 to the sciatic nerve ("pre-gp/20") and 2 weeks after, prior to topical application ("predrug"), were measured. Rats were tested within I minute ("immediate") of removal of the gauze pad, and 5 and 10 minutes following removal of the gauze pad. Data are presented as mean \pm standard error of the mean ( $n=7-10$ animals per treatment group). (B) Percent changes in thermal withdrawal latencies following topical mepivacaine treatment, calculated from predrug baseline scores in (A). (C) Percent of animals in each dose group reaching $\geq 33 \%$ increase in thermal withdrawal latencies from predrug baselines. $* P<0.05, * * P<0.01$, and $* * * P<0.00$ I vs vehicle-treated rats.

emergence of pressure and heat hyperalgesia, respectively, 2 weeks following epineural gp120 administration.

Topical administration of mepivacaine produced significant antinociception (overall $F[d f 5,4]=59.5, P<0.001$ ) as indicated by an increase in withdrawal threshold (Figure 1). By contrast, vehicle treatment alone did not significantly alter withdrawal thresholds. The antinociceptive effects of mepivacaine on gp120-induced mechanical hyperalgesia were most robust immediately following removal of the topical bandage, and tended to dissipate over the next 5-10 minutes. Significant antinociceptive effects were achieved by mepivacaine concentrations $4,8,16$, and $32 \mathrm{mM}(P<0.001$ compared with vehicle treatment). No significant reversal of pressure hyperalgesia was observed with the lowest tested mepivacaine concentration $(2 \mathrm{mM})$. Antinociceptive effects were sustained for at least 5 minutes following removal of the adhesive containing the two highest concentrations of mepivacaine ( $P<0.05$ and $P<0.001$ for 16 and $32 \mathrm{mM}$, respectively).

Results are shown as percent change from predrug baselines in Figure 1B. Using this evaluation, all concentrations of mepivacaine appeared to increase mechanical thresholds from baseline, although statistically significant increases from individual baseline responses were reached only immediately following gauze pad removal $(P<0.001$ at 4 and $8 \mathrm{mM}$ concentrations and $P<0.01$ at $16 \mathrm{mM}$ concentration compared with vehicle) or at 5 minutes $(P<0.05$ at 8 and $32 \mathrm{mM}$ concentrations compared with vehicle).

Data were also evaluated using a 33\% "analgesia" criterion across all subjects in a dosing group, since this is frequently considered as indicative of clinically relevant efficacy. ${ }^{2,5}$ According to this analysis, $50 \%-90 \%$ of the topical mepivacaine-treated animals tested displayed clinically significant antinociception at any given time point. In contrast, $0 \%-10 \%$ of vehicle-treated animals showed antinociception at any given time point (Figure 1C). In this case, the antinociceptive effects of topical mepivacaine were strongest immediately at removal of the application gauze but continued for at least 5 minutes for all mepivacaine concentrations except for the lowest ( $2 \mathrm{mM})$.

Topical mepivacaine treatment significantly increased withdrawal latencies as assessed by the heat hyperalgesia test (Figure 2; overall $F[d f 5,4]=7.4, P<0.001$ ). Similar to responses of decreased withdrawal thresholds to noxious pressure, withdrawal response latencies to noxious heat were decreased 2 weeks following sciatic nerve exposure to gp120, indicative of thermal hyperalgesia (pre-gp120 vs predrug responses). In animals that received topical mepivacaine, the most robust antinociceptive response was observed 5 minutes following gauze removal $(P<0.001$ for the 8,16 , and $32 \mathrm{mM}$ concentrations and $P<0.05$ for the $4 \mathrm{mM}$ concentration, compared with vehicle). The highest concentration (32 mM) showed antinociceptive effects beginning immediately following pad removal $(P<0.01$ compared with vehicle). As observed for mechanical hyperalgesia, antinociceptive effects 
of topical mepivacaine dissipated by $\sim 10$ minutes following removal of the gauze. In contrast, vehicle application to the hind paw did not affect thermal hyperalgesia at any given time point.

The percent change from predrug baseline following treatment is shown in Figure 2B. While all concentrations of mepivacaine produced positive increases in withdrawal latencies, statistically significant differences between treatment groups were not found ( $P>0.05$ compared with vehicle). None of the mepivacaine concentrations showed a $>50 \%$ change from baseline. (By contrast, in the paw pressure test, neuropathic rats treated with either 16 or $32 \mathrm{mM}$ of mepivacaine showed $>50 \%$ changes from baseline [Figure 1B].)

When the percent of animals reaching $33 \%$ or better "analgesia" was analyzed, $20 \%-60 \%$ of animals that received mepivacaine concentration in the 4-32 mM concentration range were found to satisfy this $33 \%$ "analgesia" criterion immediately following removal of the gauze pad (Figure $2 \mathrm{C}$ ). However, 5 minutes after removal of the gauze pad, 50\%-70\% of the rats treated with 4-32 mM mepivacaine demonstrated analgesia.

\section{Effects of topical lidocaine on gp I20- induced neuropathic hyperalgesia}

In neuropathic rats only modest and transient antinociceptive effects of lidocaine on pressure hyperalgesia were observed $(P<0.05$ for 16 and $32 \mathrm{mM}$ lidocaine compared with vehicle, immediately following gauze pad removal) (Figure 3A). When calculated as a percent change from baseline, no significant differences between lidocaine treatment and vehicle were observed (Figure 3B). In addition, only $15 \%-40 \%$ animals reached the $33 \%$ analgesia criterion following topical lidocaine treatment in the 4-32 mM range (Figure 3C).

Topical lidocaine appeared more effective against thermal hyperalgesia, with 8 and $32 \mathrm{mM}$ concentrations producing significantly increased thermal withdrawal latencies immediately following gauze removal $(P<0.01$ and 0.05 , respectively, for 32 and $8 \mathrm{mM}$, compared with vehicle, Figure 4A). This is also suggested by analyzing percent change from baseline (Figure 4B), with some treatments resulting in 70\%-80\% increases in thermal response latencies from baseline, although these were not statistically significant from vehicle. In addition, $30 \%-70 \%$ of animals reached the $33 \%$ analgesia criterion (Figure 4C). Topical lidocaine in this concentration range appeared to sustain antinociceptive effects on thermal hyperalgesia for up to 10 minutes following gauze pad removal (Figure $4 \mathrm{~A}-\mathrm{C}$ ).
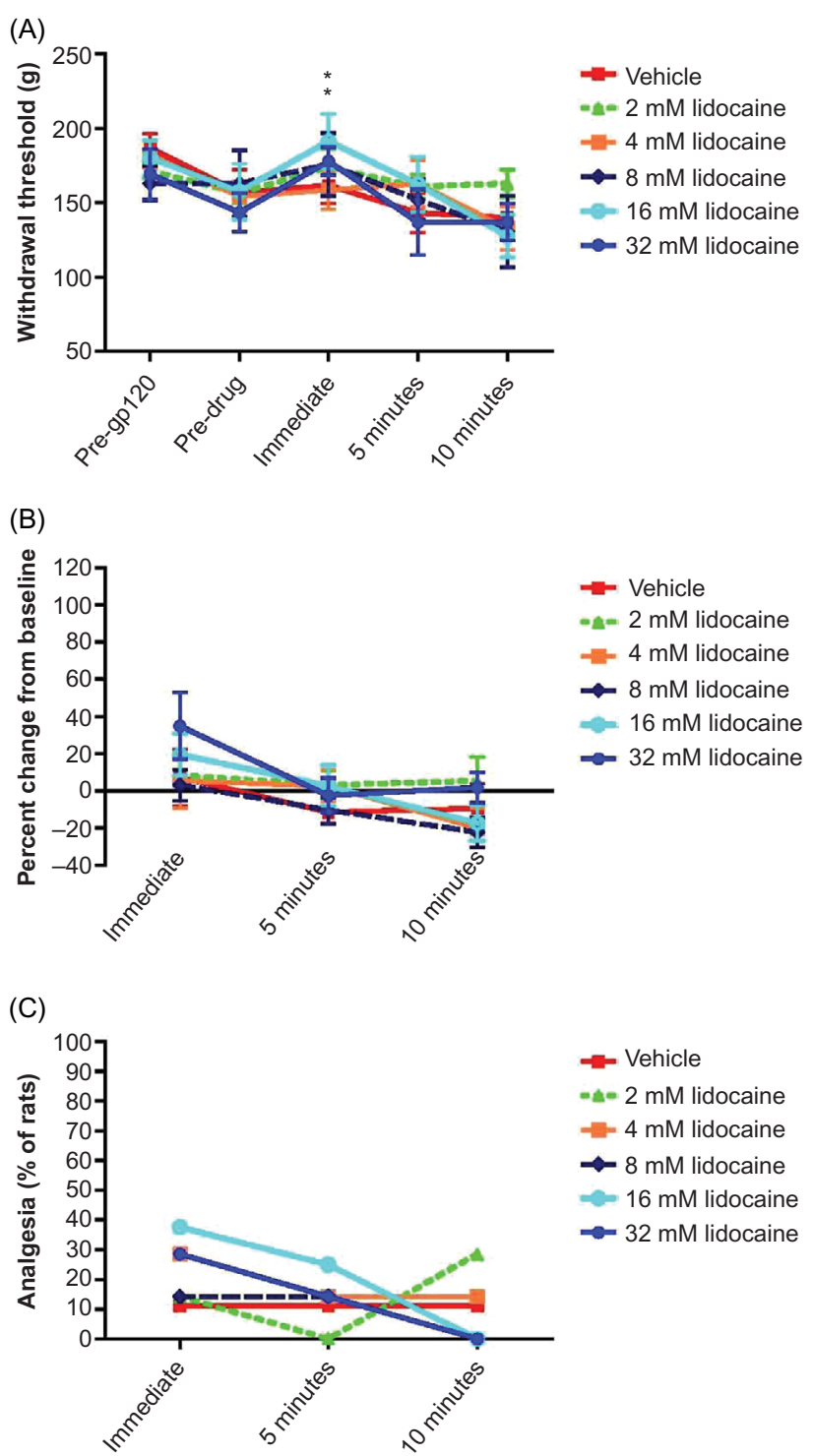

Figure 3 Effect of topically applied lidocaine on mechanical hyperalgesia in rats with gPl20-induced neuropathic pain.

Notes: (A) Effects of several concentrations (in millimolars) of topical lidocaine or vehicle on hind paw withdrawal thresholds (in grams). Withdrawal thresholds before application of gp/20 to the sciatic nerve ("pre-gp/20") and 2 weeks after, prior to topical application ("predrug"), were measured. Rats were tested within I minute ("immediate") of removal of the gauze pad, and 5 and 10 minutes following removal of the gauze pad. Data are presented as mean \pm standard error of the mean ( $n=7-10$ animals per treatment group). (B) Percent changes in mechanical withdrawal thresholds following topical lidocaine treatment, calculated from predrug baseline scores in (A). (C) Percent of animals in each dose group reaching $\geq 33 \%$ increase in mechanical withdrawal threshold from predrug baselines. ${ }^{*} P<0.05$, vs vehicle-treated rats.

\section{Effects of topical anesthetics on acute nociception in mice}

Peak antinociceptive effects of all local anesthetic concentrations except $4 \mathrm{mM}$ lidocaine $(P<0.01$ for 32 and $8 \mathrm{mM}$ mepivacaine and $P<0.05$ for $16 \mathrm{mM}$ mepivacaine and $10 \mathrm{mM}$ lidocaine compared with vehicle) were observed 5 minutes following removal of the tail from the anesthetic 

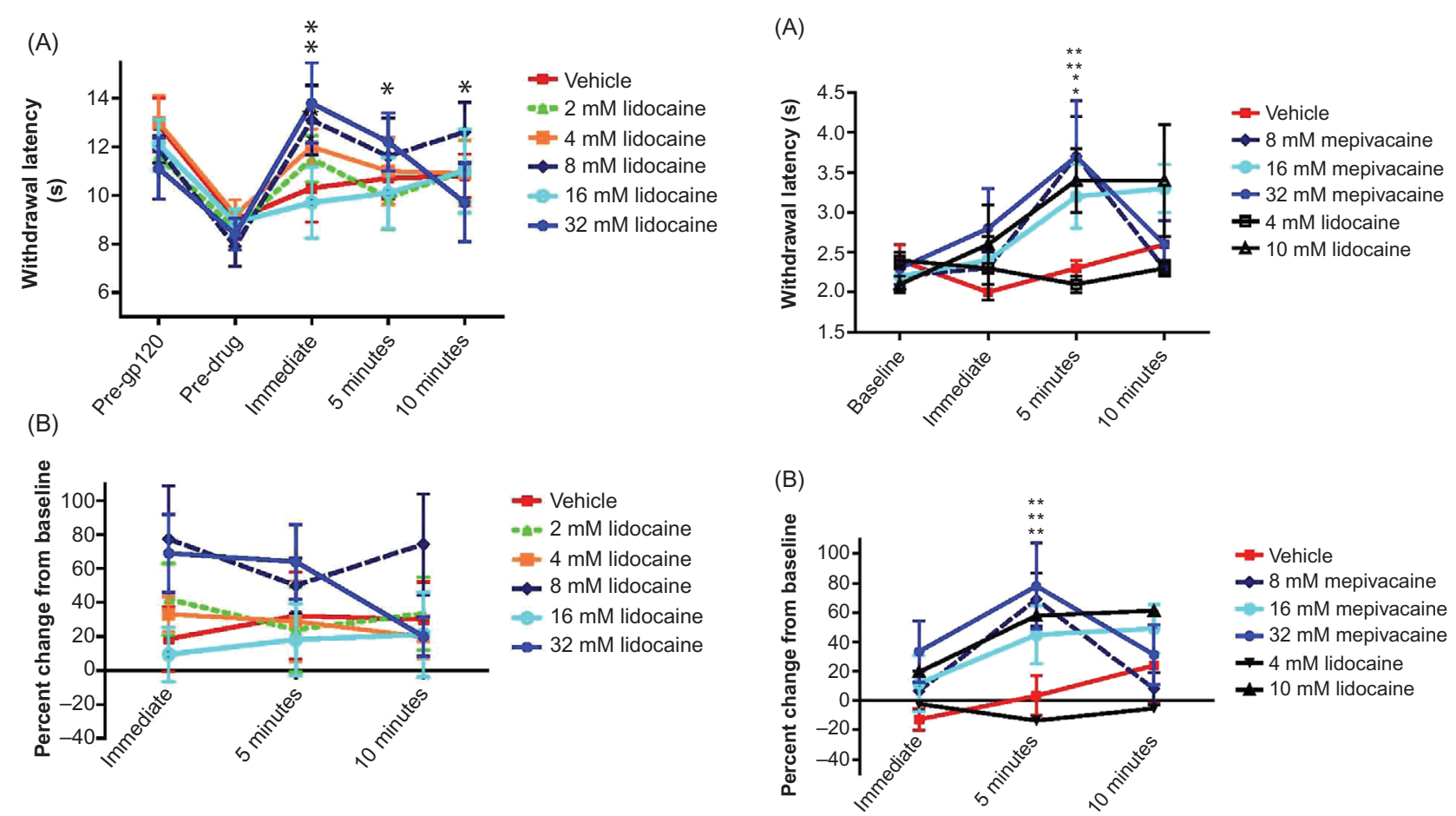

(C)

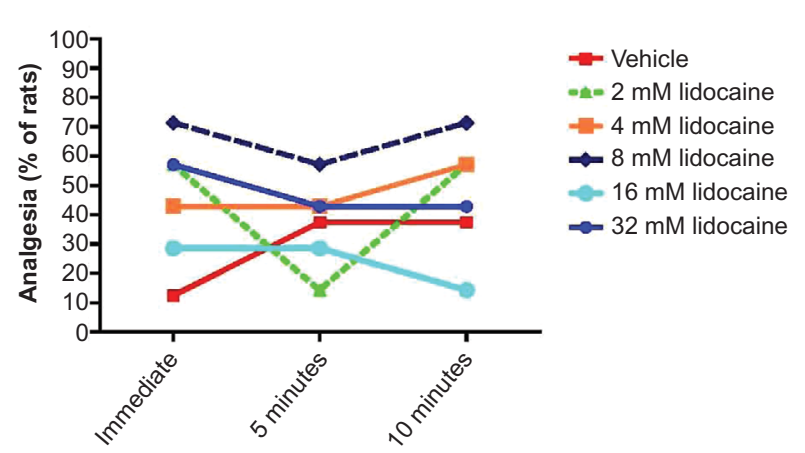

(C)

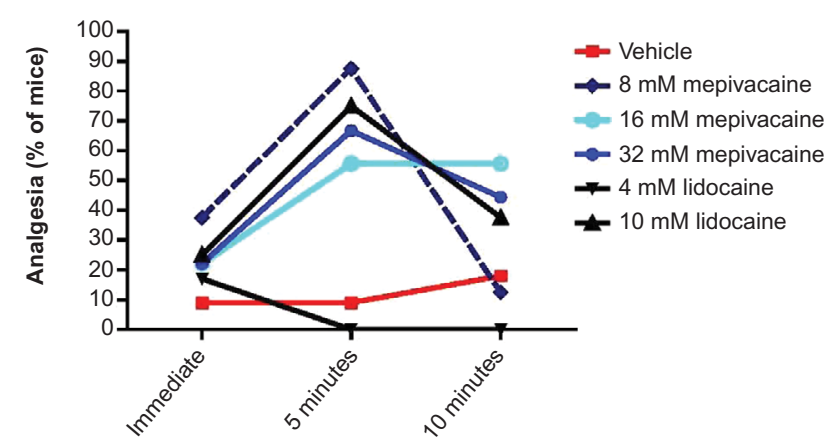

Figure 4 Effect of topically applied lidocaine on thermal hyperalgesia in rats with gp I20-induced neuropathic pain.

Notes: (A) Effects of several concentrations (in milimolars) of topical lidocaine or vehicle on hind paw withdrawal latencies (in seconds). Withdrawal latencies before application of gp/20 to the sciatic nerve ("pre-gp/20") and 2 weeks after, prior to topical application ("predrug"), were measured. Rats were tested within I minute ("immediate") of removal of the gauze pad, and 5 and 10 minutes following removal of the gauze pad. Data are presented as mean \pm standard error of the mean $(n=7-10$ animals per treatment group). (B) Percent changes in thermal withdrawal latencies following topical lidocaine treatment, calculated from predrug baseline scores in (A). (C) Percent of animals in each dose group reaching $\geq 33 \%$ increase in thermal withdrawal latencies from predrug baselines. Asterisks indicate differences between lidocaine treatment compared with saline-treated rats: $* P<0.05$.

solution (Figure 5A). Similarly, significant efficacy of these treatments was apparent when the increases in tail withdrawal latencies were converted to a percent change from baseline (Figure 5B; $P<0.01$ at 5 minutes posttreatment for $32 \mathrm{mM}$ mepivacaine, $8 \mathrm{mM}$ mepivacaine, and $10 \mathrm{mM}$ lidocaine, compared with vehicle). All concentrations of local anesthetics, except $4 \mathrm{mM}$ lidocaine, produced 50\%-90\% antinociception as assessed by the $33 \%$ analgesia criterion

Figure 5 Effects of topically applied mepivacaine and lidocaine on acute nociception in the mouse tail flick assay.

Notes: (A) Effects of several concentrations (in millimolars) of topical mepivacaine, lidocaine, or vehicle on tail flick withdrawal latencies (in seconds). Withdrawal latencies were measured before application of drug to the tail ("baseline"). Mice were tested within I minute ("immediate") of removal of the tail from the topical solution, and 5 and 10 minutes following removal of the tail from the topical solution. Data are presented as mean \pm standard error of the mean $(n=7-10$ animals per treatment group). (B) Percent changes in tail flick latencies following topical anesthetic treatment, calculated from predrug baseline scores in (A). (C) Percent of animals in each dose group reaching $\geq 33 \%$ increase in tail flick latencies from predrug baselines. $* P<0.05$, $* * P<0.01$ vs vehicle-treated mice.

5 minutes after removal from local anesthetic solution (Figure 5C).

\section{Discussion}

The current study was designed to assess potential development of the local anesthetic, mepivacaine, as a topical treatment approach for reducing HIV-related painful peripheral neuropathy. While a clinical formulation for extended or 
intermittent treatment using, for example, a patch or gel can be envisioned for future development, as a necessary first step, this study evaluated the transient acute antinociceptive effects of short-term topical application in an animal model of HIV-induced painful sensory neuropathy. Topical application of mepivacaine to the hind paw significantly alleviated both mechanical and thermal hyperalgesia in the current rat model. By contrast, the topical application of the more traditionally used local anesthetic lidocaine was effective in reducing heat hyperalgesia but only marginally effective in reducing pressure hyperalgesia in this neuropathic pain model. Topical mepivacaine and lidocaine produced comparable effects in response to noxious heat in non-injured animals (the mouse tail flick test). The current data suggest that the topical application of the local anesthetic mepivacaine could have improved effectiveness in reducing painful symptoms of HIV-induced neuropathic pain.

HIV distal sensory polyneuropathies are attributable to both the disease itself and antiretroviral treatments which exacerbate neurotoxicity. ${ }^{31}$ As combination antiretroviral therapy has markedly improved survival in HIV patients and reduced some neurotoxic complications of the treatments, the prevalence of HIV sensory neuropathy has increased with increasing survival rates, and remains high globally, estimated from $20 \%$ to over $50 \%$, with nearly half of patients experiencing pain rated as "severe". 2,5,32 Symptomatic control of HIV-associated neuropathic pain is difficult to achieve using conventional analgesic therapies and further complicated by concerns with potential substance abuse disorders in this patient population. ${ }^{2,3}$ Although several therapies have been tried for its treatment, to date, no drugs have been specifically approved for painful HIV-associated neuropathy. Small clinical trials have suggested beneficial effects compared to placebo for smoked cannabis, recombinant nerve growth factor, and topical capsaicin..$^{32-39}$ These treatments however are not without side effects such as the psychomimetic effects of cannabis, myalgia that occurs following nerve growth factor treatment, and the intense, acute pain following capsaicin application. Pharmacotherapies that are used in the management of other painful peripheral neuropathies, including the anticonvulsants lamotrigine, gabapentin, and pregabalin, and the antidepressants amitriptyline, mexiletine, and memantine, have not demonstrated substantial analgesic effects on HIV-induced neuropathic pain., ${ }^{5,7-10}$

Spontaneous peripheral nerve activity following injury or disease has been suggested to have a key role in maintaining the neuropathic pain state. ${ }^{40}$ The gp120 model for HIV-associated neuropathic pain also appears to have an inflammatory pain component. ${ }^{41}$ An inflammatory-like response was observed following application of gp120 to the sciatic nerve, with axonal swelling and endoneural vasodilation. ${ }^{17}$ The inflammatory cytokine tumor necrosis factor was found within and surrounding the nerve, suggesting the presence of infiltrating immune cells. Application of tumor necrosis factor to the sciatic nerve leads to ectopic activity in rat sciatic nerve and mechanical and thermal hyperalgesia. ${ }^{42}$ Thus, peripheral nerve spontaneous activity evoked by gp120 is one mechanism by which HIV infection could lead to neuropathic pain.

Local anesthetics are thought to attenuate spontaneous activity and ectopic discharges due to upregulated sodium channels in injured peripheral nerves, thereby leading to pain relief..$^{28,43}$ The application of lidocaine to an injured nerve can suppress ectopic activity and neuropathic pain. ${ }^{44}$ Topical local anesthetics, particularly lidocaine, have been clinically evaluated in the treatment of peripheral neuropathic pain states. ${ }^{45-48}$ Several formulations of topical lidocaine are available or in development including a lidocaine 5\% patch (Lidoderm ${ }^{\circledR} 5 \%$ ), 5\% plaster, cream mixtures (eg, EMLA), ointments, gels, and sprays. The Lidoderm ${ }^{\circledR} 5 \%$ patch is one of three US Food and Drug Administration-approved treatments for the management of postherpetic neuralgia (along with gabapentin and pregabalin). Nevertheless, the lidocaine patch provides only modest pain relief in patients with postherpetic neuralgia, ${ }^{46}$ and a recent review of randomized controlled studies concludes that there is no evidence to support the use of topical lidocaine for the treatment of neuropathic pain..$^{29}$ In the only available placebo-controlled randomized clinical trial of topical lidocaine for HIV-associated neuropathic pain, the effect of treatment did not differ significantly from placebo. ${ }^{13}$ Although the lidocaine patch has not demonstrated efficacy in well-controlled clinical trials in painful HIV-associated neuropathy, in the absence of other approved therapies, it continues to be used for this purpose.

A potential value of mepivacaine for neuropathic pain treatment is its improved bioavailability compared with lidocaine. This is supported by the current findings showing a broader range of behavioral effects on pain symptoms. Mepivacaine has an intrinsic vasoconstrictor effect which could reduce the rate at which the drug is cleared away from peripheral (skin) sites of pain generation. In addition, mepivacaine may have improved neurological safety, allowing higher doses to be applied to achieve improved analgesia. Among a series of local anesthetics, mepivacaine had the lowest neurotoxic effects on primary cultured neurons (lidocaine had the highest). ${ }^{15}$ In the current study, the topical application of mepivacaine attenuated both hind paw mechanical and thermal hyperalgesia in rats with gp120-induced peripheral neuropathy. The topically applied local anesthetics in this model likely 
suppressed pain by a transient suppression of spontaneous activity of peripheral nerves. In contrast to mepivacaine, topical lidocaine suppressed heat but not mechanical hyperalgesia. It is possible that local anesthetics do not suppress mechanical hyperalgesia as well as thermal hyperalgesia, and that a higher concentration of lidocaine could also have had an antinociceptive effect on mechanical hyperalgesia. However, neurotoxicity is likely at high concentrations of lidocaine. Lidocaine and mepivacaine were equipotent in modestly reducing responsiveness to heat in normal skin, as assessed by the mouse tail flick test, similar to the effects reported by others. ${ }^{30}$ Thus, the improved analgesic effects of topical mepivacaine, to expand coverage to include suppression of mechanical as well as heat hyperalgesia, may be indicative of distinct mechanisms such as inhibition of a wide range of nociceptor subpopulations.

There is a need for an alternative, topically applied local anesthetic for the treatment of HIV-induced painful peripheral neuropathy. Although the gp120 model produces a mononeuropathy in contrast to the distal symmetrical polyneuropathy more characteristic of HIV-associated neuropathic pain, topical treatment approaches using other agents (eg, capsaicin ${ }^{34-37}$ ) have been explored for this condition, and a potential value of topical application is the ability to apply it locally (or bilaterally) on an as-needed basis. Thus, topical anesthetics can improve patient compliance, and dosing is not a significant safety or off-target issue compared with orally or parenterally administered medications. Mepivacaine in the current study demonstrated good antinociception in a rat model of gp120-induced peripheral neuropathic pain, whereas an equivalent concentration of lidocaine that demonstrated comparable antinociception in the tail flick test was not effective on neuropathic mechanical hypersensitivity. Thus, mepivacaine could be a useful treatment in managing HIV-induced painful peripheral neuropathy.

It should be noted that, like most experimental animal models relying on assessment of evoked responses to cutaneous stimuli, the more common complaints of patients with HIV-related neuropathic pain, such as spontaneous burning and stabbing sensations, can only be inferred using these outcome measures. HIV neuropathic pain patients report a number of sensory disturbances including sensitivity to temperature and touch. ${ }^{1}$ Future preclinical efforts focused on the development of clinical therapies should include newly emerging models to capture non-evoked spontaneous pain outcomes. In addition, with the development of transgenic and HIV humanized mouse models, future studies using these models may further strengthen the search for effective therapeutics in the treatment of HIV-induced neuropathic pain.
Although significant analgesic efficacy was achieved by topical mepivacaine in this initial exploration of HIVassociated painful sensory neuropathy, a limitation in its potential therapeutic usefulness is the transient and rapidly dissipating antinociceptive effect. The development of improved transdermal delivery systems should overcome this limitation and prolong analgesic effects of topical local anesthetics. Gel formulations showing enhanced local anesthetic action in tail flick test using various agents (mepivacaine, prilocaine, bupivacaine) have been reported. ${ }^{49,50}$ Thus, future directions will include development of mepivacaine gel formulations for evaluation as a treatment approach in managing HIV-associated neuropathic pain. Based on the promising outcomes in the current study, clinical formulations of mepivacaine gels with increased permeation over time compared with 5\% lidocaine patch are being developed by the study sponsor. These advances should markedly improve the efficacy and safety in the management of neuropathic pain syndromes, particularly HIV-induced painful peripheral neuropathy.

\section{Acknowledgments}

This study was supported by Relmada Therapeutics, Inc. The authors are highly grateful to Ms Ludmilla Rusakova for her expert technical assistance.

\section{Disclosure}

Jacqueline Sagen served as a consultant to Relmada Therapeutics. The authors report no other conflicts of interest in this work.

\section{References}

1. Freeman R, Baron R, Bouhassiara D, Cabrera J, Emir B. Sensory profiles of patients with neuropathic pain based on neuropathic pain symptoms and signs. Pain. 2014;155:367-376.

2. Phillips TJC, Cherry CL, Cox S, Marshall SJ, Rice ASC. Pharmacological treatment of painful HIV-associated sensory neuropathy: a systematic review and meta-analysis of randomised controlled trials. PLoS ONE. 2010;5:e14433.

3. Robinson-Papp J, Morgello S, Vaida F, et al. Association of self-reported painful symptoms with clinical and neurophysiological signs in HIVassociated sensory neuropathy. Pain. 2010;151:732-736.

4. Robertson K, Kumwenda J, Supparatpinyo K, et al. A multinational study of neurological performance in antiretroviral therapy-naive HIV1 -infected persons in diverse resource-constrained settings. JNeurovirol. 2011;17:438-447.

5. Schütz SG, Robinson-Papp J. HIV-related neuropathy: current perspectives. HIV/AIDS (Auckl). 2013;5:243-251.

6. Verma S, Estanislao L, Simpson D. HIV-associated neuropathic pain: epidemiology, pathophysiology and management. CNS Drugs. 2005;19:325-334.

7. Kemper CA, Kent G, Burton S, Deresinski SC. Mexiletine for HIVinfected patients with painful peripheral neuropathy: a double-blind, placebo-controlled, crossover treatment trial. J Acquir Immune Defic Syndr Hum Retrovirol. 1998;19:367-372. 
8. Kieburtz K, Simpson D, Yiannoutsos C, et al. A randomized trial of amitriptyline and mexiletine for painful neuropathy in HIV infection. AIDS Clinical Trial Group 242 Protocol Team. Neurology. 1998;51:1682-1688.

9. Shlay JC, Chaloner K, Max MB, et al. Acupuncture and amitriptyline for pain due to HIV-related peripheral neuropathy: a randomized control trial. JAMA. 1998;280:1590-1595.

10. Simpson DM, Dorfman D, Olney RK, et al. Peptide T in the treatment of painful distal neuropathy associated with AIDS: results of a placebocontrolled trial. Neurology. 1996;47:1254-1259.

11. Attal N, Cruccu G, Baron R, et al; European Federation of Neurological Societies. EFNS guidelines on the pharmacological treatment of neuropathic pain: 2010 revision. Eur J Neurol. 2010;17:1113-e88.

12. Dworkin RH, O’Connor AB, Backonja M, et al. Pharmacologic management of neuropathic pain: evidence-based recommendations. Pain. 2007; 132:237-251

13. Estanislao L, Carter K, McArthur J, Olney R, Simpson D; Group L-HN. A randomized controlled trial of 5\% lidocaine gel for HIV-associated distal symmetric polyneuropathy. J Acquir Immune Defic Syndr. 2004;37:1584-1586.

14. Brockmann WG. Mepivacaine: a closer look at its properties and current utility. Gen Dent. 2014;62:70-75.

15. Radwan IA, Saito S, Goto F. The neurotoxicity of local anesthetics on growing neurons: a comparative study of lidocaine, bupivacaine, mepivacaine, and ropivacaine. Anesth Analg. 2002;94:319-324.

16. Lee HI, Park YS, Cho TG, Park SW, Kwon JT, Kim YB. Transient adverse neurologic effects of spinal pain blocks. J Korean Neurosurg Soc. 2012;52:228-233.

17. Herzberg U, Sagen J. Peripheral nerve exposure to HIV viral envelope protein gp120 induces neuropathic pain and spinal gliosis. J Neuroimmunol. 2001;116:29-39.

18. Wallace VC, Blackbeard J, Pheby T, et al. Pharmacological, behavioural and mechanistic analysis of HIV-1 gp120 induced painful neuropathy. Pain. 2007;133:47-63.

19. Apostolski S, McAlarney T, Hays AP, Latov N. Complement dependent cytotoxicity of sensory ganglion neurons mediated by the gp120 glycoprotein of HIV-1. Immunol Invest. 1994;23:47-52.

20. Milligan ED, Mehmert KK, Hinde JL, et al. Thermal hyperalgesia and mechanical allodynia produced by intrathecal administration of the human immunodeficiency virus-1 (HIV-1) envelope glycoprotein, gp120. Brain Res. 2000;861:105-116.

21. Oh SB, Tran PB, Gillard SE, Hurley RW, Hammond DL, Miller RJ. Chemokines and glycoprotein 120 produce pain hypersensitivity by directly exciting primary nociceptive neurons. $J$ Neurosci. 2001;21:5027-5035.

22. Keswani SC, Polley M, Pardo CA, Griffin JW, McArthur JC, Hoke A. Schwann cell chemokine receptors mediate HIV-1 gp120 toxicity to sensory neurons. Ann Neurol. 2003;54:287-296.

23. Jones G, Zhu Y, Silva C, et al. Peripheral nerve-derived HIV-1 is predominately CCR5-dependent and causes neuronal degeneration and neuroinflammation. Virology. 2005;334:178-193.

24. Melli G, Keswani SC, Fischer A, Chen W, Hoke A. Spatially distinct and functionally independent mechanisms of axonal degeneration in a model of HIV-associated sensory neuropathy. Brain. 2006;129:1330-1338.

25. Babul N, Hama A, Basler A, Baum J, Sagen J. Response to the local anesthetic TQ-1019 in the GP120 model of painful HIV neuropathy. In: 12th World Congress on Pain; Aug, 17-24, 2012; Glasgow, Scotland. Abstract PW350.

26. Rusakova L, Babul N, Castellanos D, Weinrich J, Baum D, Sagen J. Antinociceptive response of the local anesthetic TQ-1019 in the radiant heat tail flick assay. In: 12th World Congress on Pain; Aug, 17-24, 2012; Glasgow, Scotland. Abstract PW353.

27. Hargreaves K, Dubner R, Brown F, Flores C, Joris J. A new and sensitive method for measuring thermal nociception in cutaneous hyperalgesia. Pain. 1988;32:77-88.
28. Anitescu M, Benzon HT, Argoff CE. Advances in topical analgesics. Curr Opin Anesthesiol. 2013;26:555-561.

29. Wiffen DS, Moore RA, Quinlan J. Topical lidocaine for neuropathic pain in adults. Cochrane Database Syst Rev. 2014;7:CD010958.

30. Kolesnikov YA, Chereshnev I, Pasternak GW. Analgesic synergy between topical lidocaine and topical opioids. J Pharmacol Exp Ther. 2000;295:546-551.

31. Ghosh RK, Ghosh SM, Chawla S. Recent advances in antiretroviral drugs. Expert Opin Pharmacother. 2011;12:31-46.

32. Ellis RJ, Toperoff W, Vaida F, et al. Smoked medicinal cannabis for neuropathic pain in HIV: a randomized, crossover clinical trial. Neuropsychopharmacology. 2009;34:672-680.

33. Abrams DI, Jay CA, Shade SB, et al. Cannabis in painful HIV-associated sensory neuropathy: a randomized placebo-controlled trial. Neurology. 2007;68:515-521.

34. Paice JA, Ferrans CE, Lashley FR, Vizgirda V, Pitrak D. Topical capsaicin in the management of HIV-associated peripheral neuropathy. J Pain Symptom Manage. 2000;19:45-52.

35. Simpson DM, Brown S, Tobias J; NGX-4010 C107 Study Group. Controlled trial of high-concentration capsaicin patch for treatment of painful HIV neuropathy. Neurology. 2008;70:2305-2313.

36. Simpson DM, Estanislao L, Brown SH, Sampson J. An open-label pilot study of high-concentration capsaicin patch in painful HIV neuropathy. J Pain Symptom Manage. 2008;35:299-306.

37. Simpson DM, Brown S, Tobias JK, Vanhove GF. NGX-4010, a capsaicin $8 \%$ dermal patch, for the treatment of painful HIV-associated distal sensory polyneuropathy. Clin J Pain. 2014;30:134-142.

38. Simpson DM, McArthur JC, Olney R, et al; Lamotrigine HIV Neuropathy Study Team. Lamotrigine for HIV-associated painful sensory neuropathies: a placebo-controlled trial. Neurology. 2003;60:1508-1514.

39. Simpson DM, Olney R, McArthur JC, Khan A, Godbold J, EbelFrommer K. A placebo-controlled trial of lamotrigine for painful HIVassociated neuropathy. Neurology. 2000;54:2115-2119.

40. Baron R. Peripheral neuropathic pain: from mechanisms to symptoms. Clin J Pain. 2000;16:S12-S20.

41. Nasirinezhad F, Jergova S, Pearson JP, Sagen J. Attenuation of persistent pain-related behavior by fatty acid amide hydrolase (FAAH) inhibitors in a rat mode of HIV sensory neuropathy. Neuropharmacology. 2015;95:100-109.

42. Sorkin LS, Xiao WH, Wagner R, Myers RR. Tumour necrosis factoralpha indices ectopic activity in nociceptive primary afferent fibers. Neuroscience. 1997;81:255-262.

43. Flores MP, Castro AP, Nascimento JS. Topical analgesics. Rev Bras Anestesiol. 2012;62:244-254.

44. Kirillova I, Teilban A, Gorodetskaya N, et al. Effect of local and intravenous lidocaine on ongoing activity in injured afferent nerve fibers. Pain. 2011;152:1562-1571.

45. Devers A, Galer BS. Topical lidocaine patch relieves a variety of neuropathic pain conditions: an open-label study. Clin J Pain. 2000;16:205-208.

46. Meier T, Wasner G, Faust M, et al. Efficacy of lidocaine patch $5 \%$ in the treatment of focal peripheral neuropathic pain syndromes: a randomized, double-blind, placebo-controlled study. Pain. 2003;16:151-158.

47. Madsen CS, Johnsen BJ, Fulglsang-Frederiksen A, Jensen TS, Finnerup NB. Differential effects of a $5 \%$ lidocaine medicated patch in peripheral nerve injury. Muscle Nerve. 2013;48:265-271.

48. Peppin JF, Albrecht PJ, Argoff C, et al. Skin matters: a review of topical treatments for chronic pain. Part two: treatments and applications. Pain Ther. 2015;4:33-50.

49. Cho CW, Choi JS, Shin SC. Enhanced local anesthetic action of mepivacaine from the bioadhesive gels. Pak J Pharm Sci. 2011;24:87-93.

50. Kang C, Shin SC. Development of prilocaine gels for enhanced local anesthetic action. Arch Pharm Res. 2012;35:1197-1204. 
The Journal of Pain Research is an international, peer reviewed, open access, online journal that welcomes laboratory and clinical findings in the fields of pain research and the prevention and management of pain. Original research, reviews, symposium reports, hypothesis formation and commentaries are all considered for publication
The manuscript management system is completely online and includes a very quick and fair peer-review system, which is all easy to use. Visit http://www.dovepress.com/testimonials.php to read real quotes from published authors. 